\title{
Endoscopic mucosal resection of a glomus tumour of the duodenum
}

\author{
Atefe Sadidoust ${ }^{1}$, Mohammad Hossein Anbardar², Kamran B. Lankarani ${ }^{3}$ \\ ${ }^{1}$ Gastroenterology and Hepatology Research Centre, Shiraz University of Medical Sciences, Shiraz, Iran \\ 2Department of Pathology, Shiraz School of Medicine, Shiraz University of Medical Sciences, Shiraz, Iran \\ ${ }^{3}$ Health Policy Research Centre, Institute of Health, Shiraz University of Medical Sciences, Shiraz, Iran
}

Gastroenterology Rev 2020; 15 (2): 173-174 DOI: https://doi.org/10.5114/pg.2020.95559

\begin{abstract}
Address for correspondence: Kamran B. Lankarani MD, Health Policy Research Centre, Institute of Health, Shiraz University of Medical Sciences, Shiraz, Iran, e-mail: kblankarani@gmail.com
\end{abstract}

Glomus tumours (GT) are proliferative lesions originating from the mesenchymal tissue of glomus bodies, which are arteriovenous shunts involved in thermoregulation of the subungual zones of the fingers and toes $[1,2]$. These tumours are rare, accounting for less than $2 \%$ of all soft-tissue tumours [2]. Glomus bodies are even rarer in the visceral organs, including the gastrointestinal tract [3]. Female predominance is seen in this tumour epidemiology, and it usually occurs in the fifth or sixth decade of life [1, 2].

The stomach is the most common site of GT in the gastrointestinal tract [4]. The most common presentation is gastrointestinal bleeding, although many cases might be discovered as an incidental finding during upper endoscopy. Endoscopically they are seen as subepithelial lesions, which may resemble carcinoid tumours or gastrointestinal stromal tumours (GIST) among others [5]. Immunohistochemistry (IHC) should be done for definite diagnosis of GT $[2,4]$. Most gastric GT are benign, but rare events of malignant transformation have been reported [4]. Although duodenal GT have been reported in the past, they are considered very rare [6].

A hypertensive and otherwise healthy 57-year-old man presented with a history of recent-onset epigastric pain and post-prandial dyspepsia. Upper GI endoscopy revealed a round subepithelial lesion in the second part of the duodenum, and endoscopic ultrasound (EUS) showed the lesion in the second part of the duodenum, measuring about $12 \times 7 \mathrm{~mm}$ with mixed echo appearance, originating from submucosa, with well-defined borders, and without penetrating to other layers. No surrounding lymph node was detected.

Endoscopic mucosal resection was done by applying a rubber band at the base of the lesion and resection with a hot snare. The resected tissue was retrieved with a basket. Histological evaluation of the lesion showed a round submucosal mass consisting of dilated blood vessels lined by normal endothelial cells and surrounded by a solid proliferation of round cells with perfectly round nuclei and acidophilic cytoplasm. There was no mitosis or atypia (Figure 1). Immunohistochemically, the duodenal tumour cells stained positive for smooth muscle actin (SMA) and vimentin and were negative for chromogranin, C-kit, desmin, cytokeratin, HMB45, Melan-A, and S100, with low proliferative index confirming the diagnosis of GT of the duodenum.

Post-resection clinical and endoscopic evaluation at $6,12,24$, and 48 months did not show any evidence of tumour recurrence.

The patient has provided informed consent for publication of the case.

This report describes a very rare case of duodenal GT presenting with dyspepsia. The diagnosis was confirmed after endoscopic resection with histology and $\mathrm{IHC}$ staining.

GT are uncommon soft-tissue tumours comprising less than $2 \%$ of all soft tissue neoplasms [2]. These tumours originate from smooth muscle cells of the glomus bodies, which are modified arterio-venous structures, which play a role in thermoregulation [7].

GT in the gastrointestinal tract is rare, and most cases are reported in the stomach [1, 2, 4]. Presenting symptoms are variable and include from total asymptomatic to upper or lower gastrointestinal bleeding, anaemia, epigastric discomfort, nausea, and vomiting $[3,4]$. Duodenal GT may present similarly, with bleeding or signs of obstruction, or may be an incidental finding on endoscopy $[8,9]$.

Computed tomography and EUS may help in the characterisation of these lesions [5]. Gastrointestinal GT 

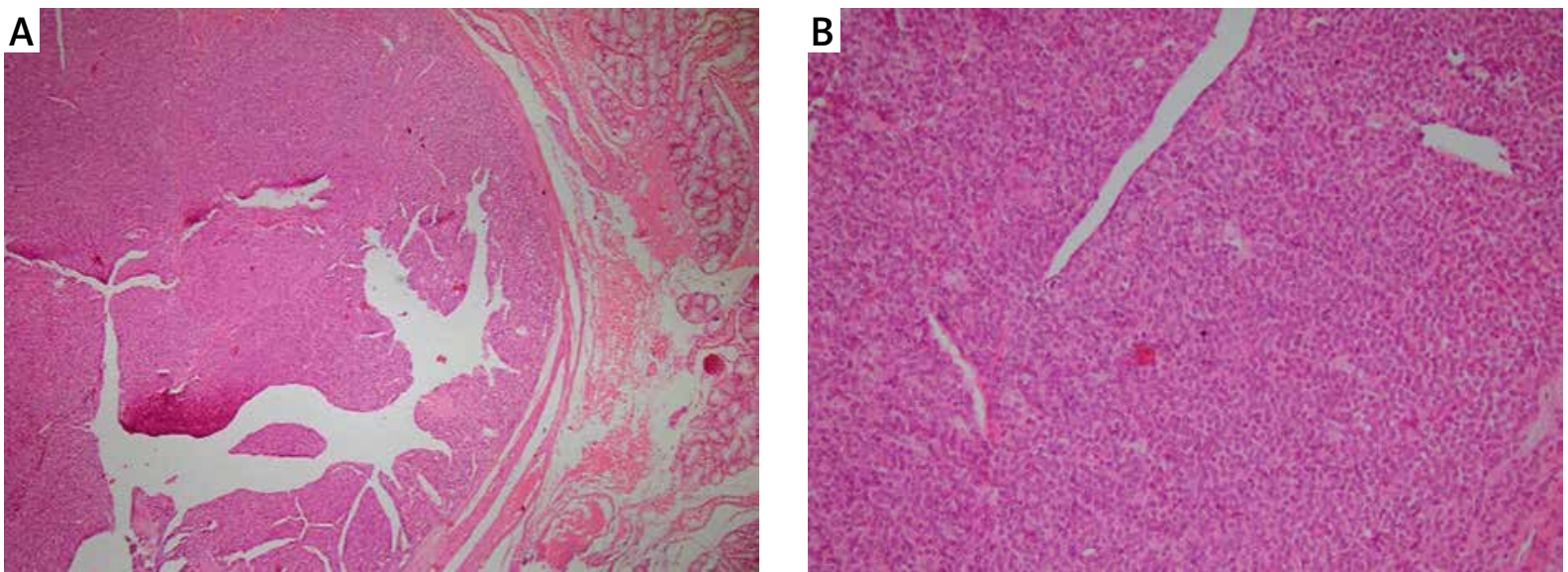

Figure 1. Histology of the resected lesion. A - Haematoxylin-eosin, original magnification 100x. B - Haematoxylin-eosin, original magnification $200 x$

should be differentiated from other tumours, including GIST, carcinoid tumours, hemangiopericytomas, paragangliomas, and lymphomas, which may have similar appearance to GT [5].

There are no specific clinical and imaging findings for GT, which makes the definite diagnosis difficult. IHC is the most reliable diagnostic tool. Morphologically the cells are small, uniform, and round with surrounding capillaries [10]. On IHC they stain positive for SMA, vimentin, calponin, collagen type IV, and laminin [2].

Surgical treatment of gastrointestinal GT consists of wedge resection or excision of the tumour, depending on the location and size $[10,11]$. There is now growing evidence that endoscopic resection of GT is possible, and in expert centres it would be a safe method. Endoscopic submucosal dissection is the preferred treatment in centres with the greatest experience in gastric GT [12]. In this case we used band ligation and a hot snare without injection to resect the lesion. This technique was reported to be safe for removal of lesions up to $20 \mathrm{~mm}$ from the gastrointestinal tract [13]. We retrieved the tissue with a basket and could have a complete resection confirmed with histologic examination. Four years after resection there was no recurrence on endoscopy or EUS.

In conclusion, GT of the duodenum should be considered in the differential diagnosis of subepithelial lesions in this region. They could be treated safely with endoscopic techniques.

\section{Conflict of interest}

The authors declare no conflict of interest.

\section{References}

1. Tsuneyoshi M, Enjoji M. Glomus tumor: a clinicopathologic and electron microscopic study. Cancer 1982; 50: 1601-7.
2. Mravic M, LaChaud G, Nguyen A, et al. Clinical and histopathological diagnosis of glomus tumor: an institutional experience of 138 cases. Int J Surg Pathol 2015; 23: 181-8.

3. Lorber J, Kalish J, Farraye FA, et al. Glomus tumor of the gastric antrum: case report. Curr Surg 2005; 4: 436-8.

4. Miettinen M, Paal E, Lasota J, Sobin LH. Gastrointestinal glomus tumors: a clinicopathologic, immunohistochemical, and molecular genetic study of 32 cases. Am J Surg Pathol 2002; 26: 301-11.

5. Oh JY, Nam KJ, Choi JC, et al. Benign submucosal lesions of the stomach and duodenum: imaging characteristics with endoscopic and pathologic correlation. Eur J Radiol 2008; 67: 112-24.

6. Portnoĭ L, Gracheva K, Kriuchkova G, Maǐskiĩ V. Glomic tumor of the duodenum. Arkhiv Patologii 1975; 37: 73.

7. Stewart DR, Sloan JL, Yao L, et al. Diagnosis, management, and complications of glomus tumours of the digits in neurofibromatosis type 1. J Med Genet 2010; 47: 525-32.

8. Chen KM, Chang MH, Lin CC. A Duodenal tumor with intermittent obstruction. Gastroenterology 2014; 146: e7-8.

9. Knackstedt C, Wasmuth H, Donner A, et al. Diagnosis of an unusual tumor in the duodenum. Endoscopy 2007; 39 (Suppl 1): E94.

10. Wu M, Zhou T, Cao D, et al. Glomus tumor of the stomach: a case report. Medicine 2018; 97: e13132.

11. Tarangelo NP, Ha K, Skole KS. Duodenal glomus tumor: a rare cause of upper gi bleeding. Clin Gastroenterol Hepatol 2016; 14: e123-4.

12. Longcroft-Wheaton G, Bhandari P. Endoscopic resection of submucosal tumors. Exp Rev Gastroenterol Hepatol 2015; 9: 659-69.

13. Faulx AL, Kothari S, Acosta RD, et al. The role of endoscopy in subepithelial lesions of the GI tract. Gastrointest Endosc 2017; 85: 1117-32.

Received: 6.09.2019

Accepted: 18.10 .2019 